\title{
A NOTE ON HILBERT'S NULLSTELLENSATZ
}

\author{
RICHARD BRAUER
}

In a recent paper, $\mathrm{O}$. Zariski ${ }^{1}$ has given a very simple proof of Hilbert's "Nullstellensatz." We give here another proof which while slightly longer is still more elementary.

Let $K$ be an algebraically closed field. We consider a system of conditions

$$
\begin{gathered}
f_{1}\left(x_{1}, x_{2}, \cdots, x_{n}\right)=0, \quad f_{2}\left(x_{1}, x_{2}, \cdots, x_{n}\right)=0, \\
\cdots, f_{r}\left(x_{1}, x_{2}, \cdots, x_{n}\right)=0 \\
g\left(x_{1}, x_{2}, \cdots, x_{n}\right) \neq 0
\end{gathered}
$$

where $f_{1}, f_{2}, \cdots, f_{r}$, and $g$ are polynomials in $n$ indeterminates $x_{1}, x_{2}$, $\cdots, x_{n}$ with coefficients in $K$. The theorem states that if the conditions (1) cannot be satisfied by any values $x_{i}$ of $K,{ }^{2}$ a suitable power of $g$ belongs to the ideal $\left(f_{1}, f_{2}, \cdots, f_{r}\right) .^{3}$

PROOF. Let $k$ be the number of $x_{j}$ which actually appear in $f_{1}, f_{2}, \cdots, f_{r}$ and let $x_{i}$ be the $x_{j}$ of this kind with the smallest subscript. Denote by $l$ the number of $f_{p}$ in which $x_{i}$ actually appears. Let $m$ be the smallest positive value which occurs as degree in $x_{i}$ of one of the $f_{\rho}{ }^{4}$ Now define a partial order for the different systems (1) using a lexicographical arrangement. If $\left(1^{*}\right)$ is a second system of the same type as (1) and if $k^{*}, l^{*}$, and $m^{*}$ have the corresponding significance, we shall say that $\left(1^{*}\right)$ is lower than (1) if either $k^{*}<k$, or $k^{*}=k$ and $l^{*}<l$, or $k^{*}=k, l^{*}=l$, and $m^{*}<m$.

Suppose now that Hilbert's theorem is false. Then there exist systems (1) which are not satisfied by any values $x_{j}$ in $K$, and for which no power of $g$ lies in $\left(f_{1}, f_{2}, \cdots, f_{r}\right)$. Choose such a system (1) taking it as low as possible. Then for all systems $\left(1^{*}\right)$ lower than (1) the theorem will hold.

If $k, l, m$ have the same significance as above, one of the $f_{\rho}$, say

Received by the editors November 1, 1947.

1 Bull. Amer. Math. Soc. vol. 53 (1947) pp. 362-368.

${ }^{2}$ If we wish to formulate the theorem for arbitrary fields $K$ as it is done in Zariski's paper, we have to consider a system of values $x_{1}, x_{2}, \cdots, x_{n}$ belonging to extension fields of finite degree over $K$. If no such system satisfies the conditions (1), the same conclusion can be drawn. The same proof can be used.

${ }^{3}$ We do not use anything from the theory of ideals except the notation $\left(f_{1}, f_{2}, \cdots, f_{r}\right)$ for the set of all polynomials of the form $P_{1} f_{1}+P_{2} f_{2}+\cdots+P_{r} f_{r}$, $P_{1} \in K\left[x_{1}, x_{2}, \cdots, x_{n}\right]$, and facts which are immediate consequences.

The numbers $k, l, m$ do not depend on $g$. 
$f_{1}$, has degree $m$ in $x_{i}$. Set

$$
f_{1}=h x_{i}^{m}+f_{1}^{*}
$$

where $h$ is the highest coefficient of $f_{1}$ as polynomial in $x_{i}$.

Neither of the following systems:

$$
\begin{aligned}
& f_{1}=0, f_{2}=0, \cdots, f_{r}=0, h=0 ; g \neq 0 ; \\
& f_{1}=0, f_{2}=0, \cdots, f_{r}=0 ; h g \neq 0
\end{aligned}
$$

can be satisfied by values $x_{j}$ of $K$, since otherwise (1) would be satisfied by the same values. Replace (3) by

$$
f_{1}^{*}=0, f_{2}=0, \cdots, f_{r}=0, h=0 ; g \neq 0 .
$$

Then $\left(3^{*}\right)$ too cannot be satisfied by values $x_{j}$ in $K$. Clearly, $\left(3^{*}\right)$ is lower than (1). Since Hilbert's theorem then holds for $\left(3^{*}\right)$, we have

$$
g^{s} \in\left(f_{1}, f_{2}, \cdots, f_{r}, h\right)
$$

for a suitable exponent $s$.

In the discussion of (4), we distinguish two cases.

Case A. $l \geqq 2$. Then $x_{i}$ appears in some $f_{\rho}$ with $\rho \geqq 2$, say in $f_{2}$. Divide $f_{2}$ by $f_{1}$ considering both as polynomials in $x_{i}$ alone. If we multiply by a suitable power $h^{a}$ of the highest coefficient $h$ of $f_{1}$, we can remove the denominators and set

$$
h^{q} f_{2}=Q f_{1}+R
$$

where $Q$ and $R$ are polynomials in all the $x_{j}$ and where $R$ is of degree smaller than $m$ in $x_{i}$. The system.

$$
f_{1}=0, R=0, f_{3}=0, \cdots, f_{r}=0 ; h g \neq 0
$$

cannot be satisfied by any values $x_{j}$ in $K$, since $\left(4^{*}\right)$ would imply (4). But $\left(4^{*}\right)$ is lower than (1) and hence Hilbert's theorem holds for $\left(4^{*}\right)$. Then, for a suitable exponent $t,(h g)^{t} \in\left(f_{1}, R, f_{3}, \cdots, f_{r}\right)$. Replacing $R$ by $h^{a} f_{2}-Q f_{1}$, we obtain

$$
h^{t} g^{t} \in\left(f_{1}, f_{2}, \cdots, f_{r}\right) .
$$

It follows from (5) that $g^{t+s t}$ belongs to

$$
g^{t}\left(f_{1}, f_{2}, \cdots, f_{r}, h\right)^{t} \subseteq g^{t}\left(f_{1}, f_{2}, \cdots, f_{r}, h^{t}\right) \subseteq\left(f_{1}, f_{2}, \cdots, f_{r}, g^{t} h^{t}\right) .
$$

Then (6) shows that $g^{t+s t} \in\left(f_{1}, f_{2}, \cdots, f_{r}\right)$, in contradiction to the assumption that no power of $g$ belongs to $\left(f_{1}, f_{2}, \cdots, f_{r}\right)$.

Case B. $l=1$. If we succeed again in establishing (6), we have the same contradiction as in the Case A, and Hilbert's theorem will be proved. 
In this case divide $g^{m+1}$ by $f_{1}$, considering both as polynomials in $x_{i}$ alone. We may then set

$$
h^{q} g^{m+1}=Q f_{1}+R
$$

where $q$ is again a positive integer, where $Q$ and $R$ are polynomials in all the $x_{j}$, and where the degree of $R$ in $x_{i}$ is smaller than $m$. Consider here the system

$$
f_{2}=0, f_{3}=0, \cdots, f_{r}=0 ; h R \neq 0.5
$$

We wish to show that $\left(4^{* *}\right)$ cannot be satisfied by values $x_{j}$ in $K$. If this were not so, choose a system of values $x_{1}^{*}, x_{2}^{*}, \cdots, x_{n}^{*}$ of $K$ which satisfy the conditions $\left(4^{* *}\right)$. Replace here $x_{i}^{*}$ by an indeterminate $x_{i}$, leaving all the other $x_{j}^{*}$ fixed. The conditions $f_{2}=0$, $f_{3}=0, \cdots, f_{r}=0$, and $h \neq 0$ are not affected, since $x_{i}$ does not appear in them. As shown by (2), the equation $f_{1}=0$ is of degree $m$ in $x_{i}$ and has therefore $m$ roots $x_{i}^{(\mu)}$ in the algebraically closed field $K$. If $g$ would not vanish when we set $x_{i}=x_{i}^{(\mu)}$, we would thus find a system of values of $K$ which satisfies all the conditions (4) and this is impossible. Hence $g$ must vanish when we set $x_{i}=x_{i}^{(\mu)}$ and it follows from (7) that the same holds for $R$. Moreover, as root of the equation $R=0$ in $x_{i}$, the quantity $x_{i}^{(\mu)}$ has the same multiplicity as for $f_{1}=0$. Thus the equation $R=0$ of degree less than $m$ in $x_{i}$ has $m$ roots $x_{i}=x_{i}^{\mu}$. Consequently, $R$ must vanish identically in $x_{i}$. However, for $x_{i}=x_{i}^{*}$, we had $R \neq 0$, as shown by $\left(4^{* *}\right)$. Thus the assumption that $\left(4^{* *}\right)$ can be satisfied by values of $K$ leads to a contradiction.

If $r>1$, the system $\left(4^{* *}\right)$ is lower than (1) and we may again apply Hilbert's theorem. This shows that a suitable power $(h R)^{v}$ belongs to $\left(f_{2}, f_{3}, \cdots, f_{r}\right)$. This still holds for $r=1$, when we interpret $\left(f_{2}, f_{3}, \cdots, f_{r}\right)$ as the zero ideal. Indeed, since $\left(4^{* *}\right)$ cannot be satisfied, $h R$ must vanish for all systems of values $x_{i}$ of $K$, and hence identically. ${ }^{6}$ Now (7) yields

$$
\left(h^{q+1} g^{m+1}\right)^{v}=\left(h Q f_{1}+h R\right)^{v} \in\left(f_{1}, f_{2}, \cdots, f_{r}\right) .
$$

If the integer $t$ satisfies the inequalities $t \geqq(q+1) v, t \geqq(m+1) v$, then (6) will hold again. But this is all we had to show and the proof of Hilbert's theorem is complete.

UNIVERSITY OF TORONTO

${ }^{5}$ If $r=1$, the system $\left(4^{* *}\right)$ is to consist only of the inequality $h R \neq 0$.

${ }^{6} \mathrm{We}$ assume the elementary theorem that if a polynomial in several variables vanishes for all systems of values of the underlying field $K$ and if $K$ is either infinite or contains at least sufficiently many elements, the polynomial vanishes identically. 